\title{
A Directional CSMA/CA Protocol for mmWave Wireless PANs
}

\author{
Michelle X. Gong, Robert Stacey, Dmitry Akhmetov \\ Intel Labs, Mobile Wireless Group \\ Intel Corporation \\ Santa Clara, CA 95054-1549 \\ Email: \{michelle.x.gong, robert.j.stacey, dmitry.akhmetov@intel.com\}
}

\author{
Shiwen Mao \\ Dept. Electrical and Computer Engineering \\ Auburn University \\ Auburn, AL 36849-5201 \\ Email: smao@ieee.org
}

\begin{abstract}
In this paper, we investigate the problem of medium access control in mmWave wireless personal area networks (WPAN), within which directional antennas are used to combat the high path loss incurred in the $60 \mathrm{GHz}$ frequency band. The conventional CSMA/CA protocol does not work well with directional antennas due to impaired carrier sensing at the transmitters. We explain why existing directional CSMA/CA protocols do not work well at $60 \mathrm{GHz}$ and propose a novel directional CSMA/CA protocol designed specifically for $60 \mathrm{GHz}$ WPANs. Instead of relying on physical carrier sensing, the proposed protocol adopts virtual carrier sensing and relies on a central coordinator to distribute network allocation vector (NAV) information. Both performance analysis and simulation study show that the proposed mechanism incurs low overhead and has robust performance even when the network is heavily congested.
\end{abstract}

\section{INTRODUCTION}

In recent years, the millimeter wave (mmWave) technology has gained considerable interest from academia, industry, and standards bodies. One of the leading factors that make mmWave technology so attractive is due to the huge unlicensed bandwidth (i.e., up to $7 \mathrm{GHz}$ ) available in the $60 \mathrm{GHz}$ band in most part of world. With this huge unlicensed bandwidth, many new applications that require gigabit data rate can be easily supported. Another important factor is that the $60 \mathrm{GHz}$ regulation allows much higher effective isotropic radiated power (EIRP) compared to other existing wireless local area networks (WLANs) and wireless personal area networks (WPANs). High EIRP is required to overcome the high path loss in the $60 \mathrm{GHz}$ band.

One of the biggest challenges for $60 \mathrm{GHz}$ is its high propagation loss. The propagation loss of $60 \mathrm{GHz}$ signals in free space is $22 \mathrm{~dB}$ higher than that of $5 \mathrm{GHz}$ signals. $60 \mathrm{GHz}$ signals also suffer from high attenuation loss due to obstacles. For instance, a human body introduces at least $15 \mathrm{~dB}$ loss to $60 \mathrm{GHz}$ signals compared to only $5 \mathrm{~dB}$ loss to $5 \mathrm{GHz}$ signal. Therefore, directional antennas, such as phased antenna arrays, are required to overcome the high propagation loss. Directional transmissions should be explicitly considered in the design of MAC protocol for mmWave WPANs.

Currently, several standards have been or are being defined to achieve multi-gigabit rate for $60 \mathrm{GHz}$ WPANs. Examples include ECMA-387 [1] and IEEE 802.15.3c [2]. Both standards focus on using Time Division Multiple Access (TDMA) for data communications. Existing MAC protocols recently proposed for $60 \mathrm{GHz}$ networks are also based on TDMA [3], [4]. Because data traffic is bursty, the required medium time is often highly unpredictable. A TDMA-based MAC protocol may cause either high overhead for on-the-fly medium reservation, or under- or over-allocated medium time for individual users. Furthermore, as defined in IEEE 802.15.3 [5], a piconet coordinator (PNC) needs to schedule bandwidth requests from associated devices. Given that scheduling is computationally intensive and should be executed in realtime, it is challenging to implement such a PNC on a mobile device.

Contention-based MAC protocols, such as Carrier Sense Multiple Access with Collision Avoidance (CSMA/CA), work well with bursty traffic and operate robustly in unlicensed bands [6]. However, the conventional CSMA/CA protocol does not work well with directional antennas due to impaired carrier sensing at the transmitter. Under CSMA/CA, devices (DEVs) that see a busy medium compete for access to the medium by waiting a random number of slot times before the next attempt for transmission. Depending on the random numbers chosen by each DEV, one DEV (i.e., the one with the smallest random number) will typically gain access to the medium first. The other DEVs will detect the transmission through a carrier sense mechanism and suspend their attempts to gain channel access until the medium becomes idle again.

In $60 \mathrm{GHz}$ band systems, beamforming, both on the receive side and the transmit side, will be used to improve signal quality at the receiver. As a result of directional listening and transmitting, the signal strength could be very low at third party devices that are not involved in the current exchange, making it difficult to perform carrier sense. This is often referred to as the deafness problem. As illustrated in Fig. 1, while Node A is transmitting to and receiving from Node C, Node A's antenna beam points towards Node C. Because Node $\mathrm{B}$ cannot sense the directional transmission from Node A to Node C, Node B may keep on trying to transmit to Node A and keep on backing off with larger and larger contention window after each transmission failure. Due to the deafness problem, new mechanisms are needed to ensure that deferral by third party devices is effective in this environment.

Many contention-based MAC protocols that support directional antennas have been proposed for mobile ad hoc 


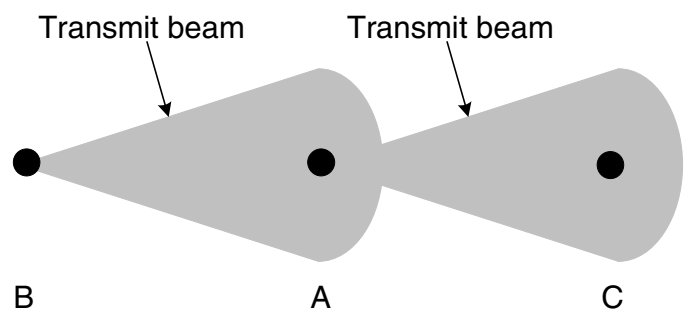

Fig. 1. Illustration of the deafness problem. Node B is deaf with regard to the directional transmission from Node A to Node C.

networks [7]-[10]. A short survey of directional MAC protocols can be found in [11]. Most of the schemes rely on RTS and/or CTS to distribute the Network Allocation Vector (NAV), which contains duration information of the subsequent transmission(s). However, most existing directional MAC protocols either are based on assumptions that do not apply to the $60 \mathrm{GHz}$ band, or introduce too much overhead when adopted in the $60 \mathrm{GHz}$ band. Section II-C elaborates on why existing directional MAC protocols cannot be directly applied in the $60 \mathrm{GHz}$ band.

In this paper, we propose a directional CSMA/CA-based medium access protocol that is tailored specifically for $60 \mathrm{GHz}$ WPANs. To the best of our knowledge, this is the first directional CSMA/CA protocol designed for $60 \mathrm{GHz}$ WPANs. The proposed protocol utilizes PNC as a central collaborator for medium access. The proposed protocol does not require scheduling and has lower protocol overhead than many schemes proposed in the literature. We derive the optimal saturation throughput and the optimal CWmin with respect to the number of simultaneous contending DEVs. Theoretical analysis and OPNET simulations both show that the proposed protocol works robustly under increased number of supported DEVs. Furthermore, the proposed protocol achieves reasonably high MAC efficiency, especially when the optimal CWmin values are chosen.

The remainder of this paper is organized as follows. We introduce the system model in Section II. The proposed MAC protocol is described in Section III. We present an analysis of the proposed MAC protocol in Section IV and a simulation study in Section V. Section VI concludes this paper.

\section{SySTEM Model}

\section{A. Network Model}

We consider a wireless personal area network as illustrated in Fig. 2. In such a network, one device (DEV) acts as a PNC that coordinates medium access for multiple other DEVs. The PNC also provides basic timing for the network and manages membership of the network. DEVs can communicate with the PNC or they can communicate with each other directly without having to bridge data through the PNC.

\section{B. Antenna Model}

Without loss of generality, we assume that each DEV is equipped with one or more directional antennas, such as steerable antennas or phased antenna array. Millimeter wave

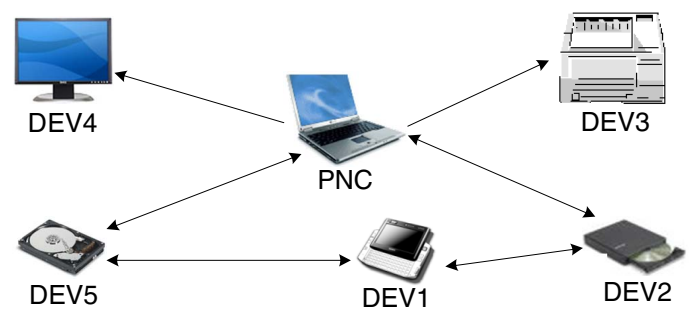

Fig. 2. A WPAN network consisting of one PNC and five DEVs.

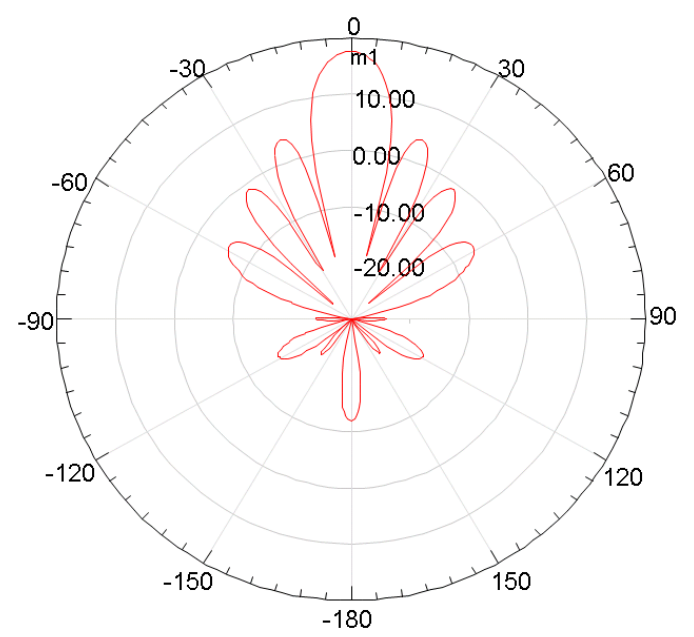

Fig. 3. Radiation pattern of an antenna array.

antennas can be made very small and multiple such antennas can be easily integrated in a chip for beamforming.

The radiation pattern of an antenna array is illustrated in Fig. 3. The beamwidth is far narrower at $60 \mathrm{GHz}$ than that at lower frequency bands, e.g., as narrow as $4.7^{\circ}$. Because there is an additional $22 \mathrm{~dB}$ propagation loss in the $60 \mathrm{GHz}$ band in free space, both ends of the link need to have directional antennas with high gains to achieve the desired data rate at the desired radio range.

\section{C. mmWave Physical Layer Model}

The challenge of $60 \mathrm{GHz}$ WPAN design arises because of the requirement for high antenna gain at both the transmitter and the receiver. However, before two devices can finish beamforming training with each other, neither one can achieve proper beam-formed transmission or reception. Therefore, a low-rate modulation and coding scheme (MCS) needs to be defined to address the case when only one end of the link has high beam-forming gain. Here, we assume a range optimized MCS, i.e. MCS0, which has about $12 \mathrm{~dB}$ higher receiver sensitivity than a data-rate-optimized MCS that offers data rates higher than $1 \mathrm{Gbps}$.

Many existing directional contention-based MAC protocols assume that normal data rate can be used even when only one end of the link uses directional antennas. This assumption is not valid anymore in the $60 \mathrm{GHz}$ band. In $60 \mathrm{GHz}$, MCS0 has to be used for beam-forming training and for any transmission when only one end of the link has high antenna gain. Further- 
more, existing directional MAC protocols assume that beamtraining or the so-called beam-locking can be performed on a single received packet. While this might be true for MIMO systems operating in lower frequency bands, such as $2.4 \mathrm{GHz}$ or $5 \mathrm{GHz}$, beam-forming training at $60 \mathrm{GHz}$ with phased arrays is much more challenging and requires multiple iterations. In a $60 \mathrm{GHz}$ system, if a DEV needs to transmit a frame such as a RTS frame in an omni-directional fashion, it needs to perform a sector sweep to transmit multiple copies of the RTS frame in different directions or sectors. While the DEV is transmitting the RTS frames, it may miss any transmission intended for itself from another DEV. Thus, none of the existing contention-based directional MAC protocols can solve the deafness problem in $60 \mathrm{GHz}$.

\section{Directional CSMA/CA Protocol}

In this section, we describe a directional CSMA/CA protocol that is designed specially for $60 \mathrm{GHz}$ WPANs. We show the proposed protocol works well for DEVs with directional antennas. It also incurs very low protocol overhead.

\section{A. Directional CSMA/CA Protocol}

As shown in Fig. 2, the WPAN network consists of one or more DEVs and a PNC. Before associating with the PNC, DEVs first perform beam-forming training for both transmission and reception such that both the transmitting and the receiving antennas can provide beam-forming gain. After a DEV completes beam-forming training, it always beam-forms towards the PNC in its idle mode (i.e., waiting to receive), meaning there is high receive antenna gain. While idle, the PNC receives in its omni mode, meaning that there is no significant receive antenna gain. In the proposed protocol, because a DEV is always beam-formed towards the PNC before any data transmission or reception and the PNC coordinates the transmission within a WPAN, the deafness problem is thus easily solved.

To describe the proposed protocol, we consider a typical scenario where DEV1 has data to transmit to DEV2. In the idle mode, both DEV1 and DEV2 are beam-formed towards the PNC. Even if DEV1 knows how to beam-form towards DEV2, DEV1 has to notify DEV2 about the impending data transmission such that DEV2 will configure its receive antennas to beam-form towards DEV1.

As shown in Fig. 4, before DEV1 can communicate with DEV2 directly, it transmits a Target Request To Send (TRTS) to the PNC. The TRTS contains three addresses: Receive Address (i.e. PNC), Transmit Address (i.e. DEV1), and Target Address (i.e. DEV2). Upon receiving the TRTS, the PNC will transmit a Target Clear To Send (TCTS) message in omni mode. This ensures that all associated DEVs can receive it. The TCTS contains three addresses: Receive Address (i.e. DEV1), Transmit Address (i.e. PNC), and Target Address (i.e. DEV2). Both TRTS and TCTS indicate the duration of the Transmission Opportunity (TXOP) and they are transmitted using MCSO because only one end of the link has beamforming gain. If the PNC does not receive the TRTS either

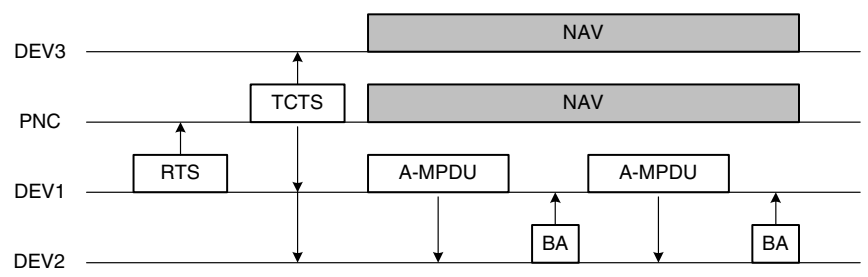

Fig. 4. Illustration of the directional CSMA/CA protocol.

due to channel error or a collision on TRTS, DEV1 will not receive a TCTS after transmitting a TRTS. DEV1 assumes that a collision has occurred and starts an exponential backoff procedure as defined in [12].

After receiving the TCTS and recognizing that its MAC address is the Target Address, DEV2 steers its beam towards DEV1. After receiving the TCTS, DEV1 steers its beam towards DEV2. The TRTS/TCTS exchange also set up a transmission opportunity (TXOP) in the network. Within the TXOP, DEV1 can transmit one or more Aggregated MAC Protocol Data Units (A-MPDUs) to DEV2 at a high data rate. Up to 64 MPDUs may be aggregated in one A-MPDU, which has a maximum size limit of $64 \mathrm{~K}$. Upon receiving an A-MPDU, DEV2 replies with a Block ACK (BA) that identifies which MPDUs in the A-MPDU have been received successfully. Other devices, such as DEV3, learn from the TCTS that there will be an on-going transmission and thus set their NAVs for the duration of the TXOP indicated in the TCTS. Note that DEV1 and DEV2 have to obey the TXOP duration and should not transmit beyond the TXOP boundary that was defined by the TRTS/TCTS exchange.

\section{B. Remarks}

Even though the proposed directional MAC protocol bears similarities with 802.11 DCF [12], there are a few important differences that are worth noting. First, DCF is a distributed MAC protocol, meaning any DEV can transmit an RTS to any other DEV. On the other hand, the proposed directional CSMA/CA protocol is a centralized protocol. Before any DEV can transmit data to other DEVs, it must transmit a TRTS to the PNC to reserve medium time. TRTS and TCTS are control frames that carry three addresses, one of which identifies the destination DEV. Due to antenna directionality, DEVs adopting a distributed MAC protocol need to continuously track all neighboring DEVs, which can incur prohibitively high communication overhead and high implementation complexity.

Second, to support directionality, some of the parameters in the proposed protocol are different from those defined in 802.11 DCF. For instance, aSlotTime is an important parameter in both DCF and our protocol and it is set to the time needed for any station to detect a transmission from any other station. In 802.11 DCF, aSlotTime is set to:

$$
\begin{aligned}
& \text { aSlotTime } \\
= & \text { aCCATime }+ \text { aRxTxTurnaroundTime }+ \\
& \text { aAirPropagationTime }+ \text { aM ACProcessingDelay, }
\end{aligned}
$$




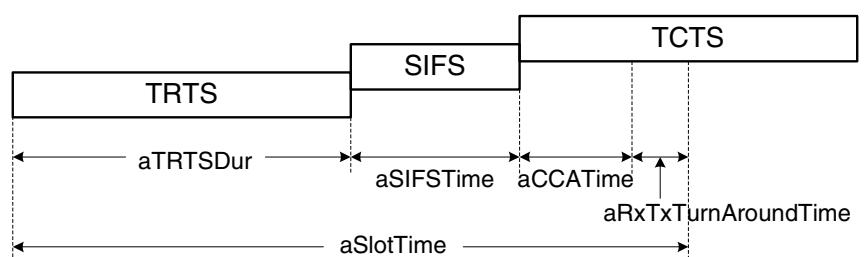

Fig. 5. Illustration of aSlotTime in the directional CSMA/CA.

whereas in our protocol, aSlotTime is set to:

$$
\begin{aligned}
& \text { aSlotTime } \\
= & \text { aTRTSDur + aSIFSTime }+ \text { aCCATime }+ \\
& \text { aRxTxTurnAroundTime. }
\end{aligned}
$$

Here, aTRTSDur is the duration of a TRTS frame, which includes the PHY preamble, the PHY header and the TRTS frame body. aSIFSTime is a short inter-frame time between receiving a packet and sending out an acknowledgement. aCCATime is the time that a receiver needs to determine whether a valid packet is on the medium. aRxTxTurnaroundTime is the time that a half-duplex device needs to switch from $\mathrm{Rx}$ mode to Tx mode.

Because all DEVs are beam-formed towards the PNC and the width of the beam generated by an antenna array is narrow, most other DEVs won't be able to receive the TRTS sent from the source DEV. Therefore, for a third-party DEV to detect an on-going transmission, virtual carrier sensing has to be used and thus aSlotTime needs to include aTRTSDur and aSIFSTime. The definition of aSlotTime in the proposed protocol is illustrated in Fig. 5.

Last but not the least, 802.11 DCF was designed mainly for omni-directional transmissions and it suffers from various problems, including the deafness problem, when being used with directional antennas. Our proposed protocol is designed specifically for directional transmissions in the $60 \mathrm{GHz}$ band and it does not incur the deafness problem.

\section{Performance Analysis}

In this section, we present an analytical study of the proposed directional MAC protocol. We derive the saturation throughput of the proposed protocol, which is defined as the throughput level achieved at the top of the MAC layer when all nodes in the systems are continuously loaded. We also investigate the optimal contention window sizes and the optimal transmission probabilities.

\section{A. Saturation Throughput}

It is assumed that devices use MAC frame aggregation schemes, such as A-MPDU, and make multiple transmissions in one TXOP. When TXOP is utilized, a device contends once to transmit TRTS. Upon successful reception of a TCTS that is addressed to itself, the device can transmit as many A-MPDU as the TXOP duration permits, provided that the last BA can be received within the TXOP duration.
We follow the assumptions made in [6] and adopt the same 2-D Markov chain model for the proposed MAC protocol. In the Markov chain mode, each state is represented by $\{s(t), b(t)\}$, where $s(t)$ is defined to be the stochastic process representing the backoff stage $(0, \cdots, m)$ of the station at time $t$ and $b(t)$ is the stochastic process representing the backoff time counter for a given station. The maximum backoff stage, i.e., $m$, takes the value such that $C W_{\max }=2^{m} C W_{\min }$, where $C W_{\max }$ is the maximum contention window and $C W_{\min }$ is the minimum contention window.

Let $S$ be the normalized system throughput, defined as the fraction of time the channel is used to successfully transmit payload bits. $S$ can be expressed as the average number of payload bits transmitted in a TXOP divided by the average length of a TXOP. Based on the 2-D Markov chain model, we extend the analysis in [6] and derive the system saturation throughput as:

$$
\begin{aligned}
S & =\frac{P_{s} P_{t r} \sum_{i=1}^{N} \mathrm{E}\left[P_{i}\right]}{\left(1-P_{t r}\right) \sigma+P_{t r} P_{s} T_{s}+P_{t r}\left(1-P_{s}\right) T_{c}} \\
T_{s} & =\sigma+a T C T S D u r+T X O P \\
T_{c} & =\sigma \\
P_{t r} & =1-(1-\tau)^{2} \\
P_{s} & =\frac{n \tau(1-\tau)^{n-1}}{1-(1-\tau)^{n}},
\end{aligned}
$$

where $T_{s}$ is the average time consumed by a successful TXOP, $T_{c}$ is the average medium time a collision consumes, $\sigma$ is the duration of a time slot, aTCTSDur is the transmission duration of the TCTS frame, $\tau$ is the probability that a device transmits in a randomly chosen time slot, $P_{s}$ is the probability that a TXOP is successfully set up, and $P_{t r}$ is the probability that there is at least one transmission in the considered slot time. The sum $\sum_{i=1}^{N} \mathrm{E}\left[P_{i}\right]$ is the combined average payload size of $N$ A-MPDUs that are transmitted in the TXOP.

Equation (1) can be rearranged as follows:

$$
S=\frac{\sum_{i=1}^{N} \mathrm{E}\left[P_{i}\right]}{T_{s}-T_{c}+\frac{T_{c}-(1-\tau)^{n}\left(T_{c}-\sigma\right)}{n \tau(1-\tau)^{n-1}}} .
$$

Under condition $\tau \ll 1, \tau$ can be estimated as [6]:

$$
\tau \approx \frac{1}{n \sqrt{T_{c} /(2 \sigma)}} .
$$

Figure 6 illustrates the relationship between the optimal saturation throughput and the number of devices in the WPAN. When the transmission probability is small, i.e., $\tau \ll 1$, the throughput degradation is small with an increase in the number of devices.

\section{B. Optimal Contention Window}

Based on the analysis in [6], the optimal contention window is derived as follows:

$$
W_{o p t}=n \sqrt{\frac{2 T_{c}}{\sigma}}=n \sqrt{2} .
$$




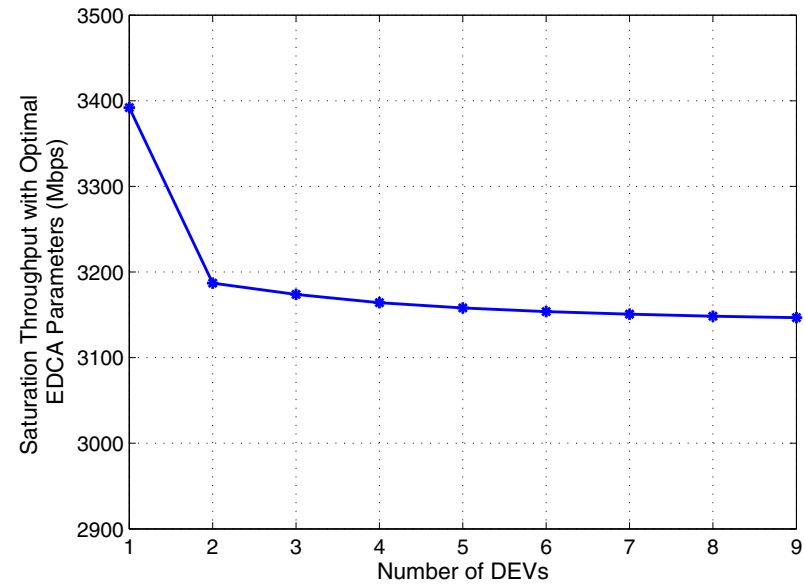

Fig. 6. Saturation throughput vs. number of devices.

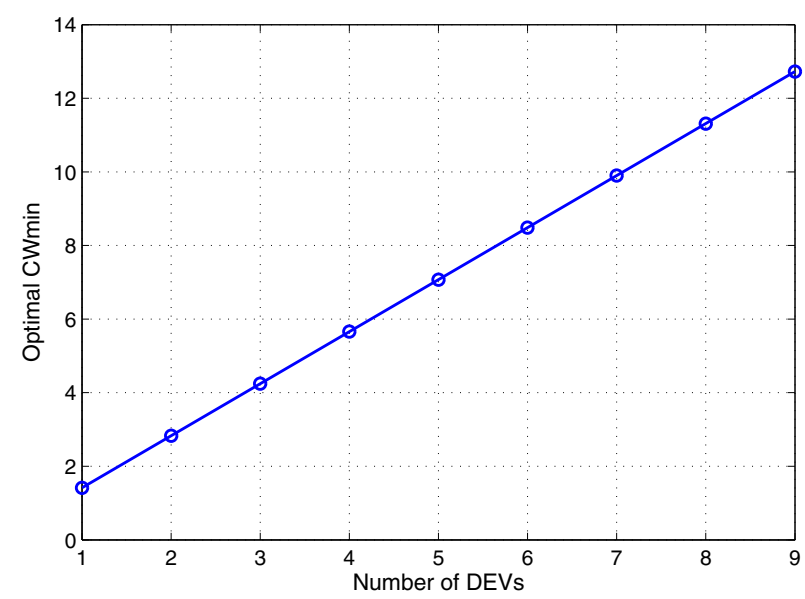

Fig. 7. Optimal CWmin vs. number of devices.

At the optimal contention window, the maximum saturation throughput can be approximated as:

$$
S_{\max }=\frac{\sum_{i=1}^{N} \mathrm{E}\left[P_{i}\right]}{T_{s}+\sigma K+T_{c}\left[K\left(e^{1 / K}-1\right)-1\right]},
$$

where $K=\sqrt{T_{c} /(2 \sigma)}$.

Note that when the optimal contention window is adopted, the maximum saturation throughput is independent of the number of DEVs. However the optimal contention window itself varies with the number of DEVs. As illustrated in Fig. 7, the optimal contention window size increases linearly with the number of contending DEVs.

\section{Transmission Probability}

The closed-form expression Eqn. (6) also allow us to investigate the impact of transmission probabilities. Fig. 8 illustrates the relationship between transmission probability and saturation throughput. When the number of devices is small, such as five, the saturation throughput degrades only slightly with an increase in the transmission probability. Due to the small collision cost of TRTS frames, when there are a small

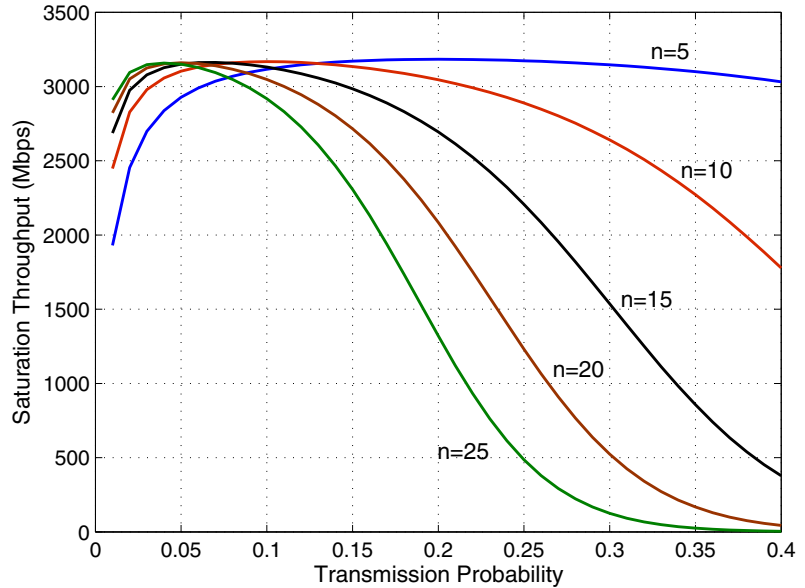

Fig. 8. Saturation throughput vs. transmission probability.

TABLE I

SimUlation PARAMETERS

\begin{tabular}{|l|l||l|l|}
\hline Parameter & Value & Parameter & Value \\
\hline \hline MCS0 Rate (Mbps) & 25 & aSlotTime (us) & 20 \\
\hline Data Rate (Mbps) & 3800 & aSIFSTime (us) & 3 \\
\hline ACK Rate (Mbps) & 1650 & TXOP duration (us) & 500 \\
\hline A-MPDU size (byte) & 65536 & MCS0 preamble (us) & 3.75 \\
\hline TRTS (byte) & 26 & Data preamble (us) & 1.75 \\
\hline TCTS (byte) & 26 & Default CWmin & 15 \\
\hline BA size (byte) & 32 & Default CWmax & 1023 \\
\hline
\end{tabular}

number of devices in the network, the throughput does not degrade even if DEVs access the medium more aggressively.

\section{Simulation Study}

We evaluate the performance of the proposed directional MAC protocol via extensive simulations using the OPNET Modeler. Our simulation uses a typical wireless PAN topology with one PNC and a variable number of devices in the same WPAN, as shown in Fig. 2. Each DEV is fully loaded with upstream traffic destined for the PNC. The simulation parameters and their values are given in Table I. The simulation results are presented in Figs. 9, 10, and 11. Each point in the figures is the average of 13 simulation runs, with $95 \%$ confidence intervals plotted (which are negligible in all cases).

As illustrated in Fig 9, when the optimal CWmin is always chosen, the throughput degrades gracefully with an increase in the number of devices. When the default $802.11 \mathrm{CWmin}$ and CWmax values are used, the simulated MAC throughput increases initially when the number of DEVs increases. When there are more than 6 DEVs in the network, the MAC throughput decreases gradually with the increase in the number of DEVs. Furthermore, the performance gap between the optimal case and the default case decreases monotonically with an increase in the number of DEVs. The reason is that for different number of DEVs, the optimal values for CWmin are different. The 802.11 default parameters, i.e. $\mathrm{CWmin}=15$ and $\mathrm{CWmax}=1023$, are optimal only when there are about 12 


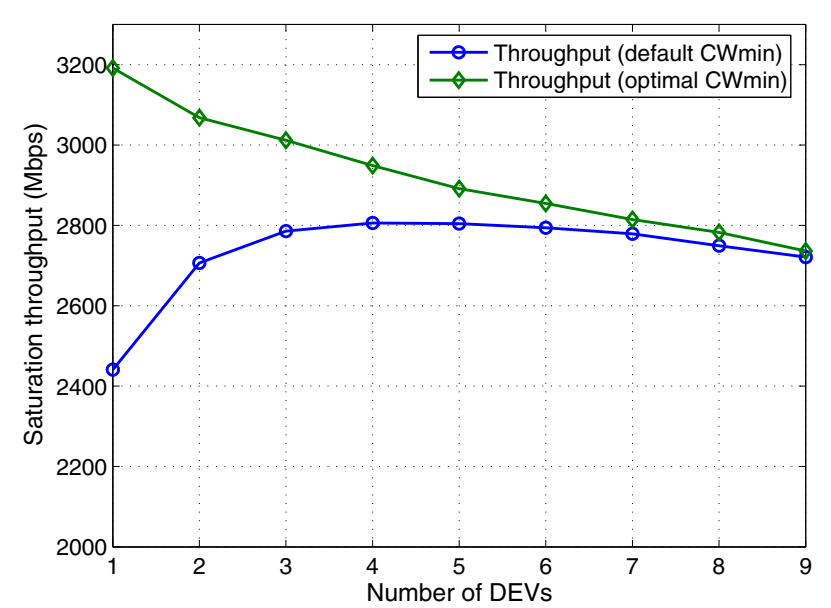

Fig. 9. Simulated throughput vs. number of devices.

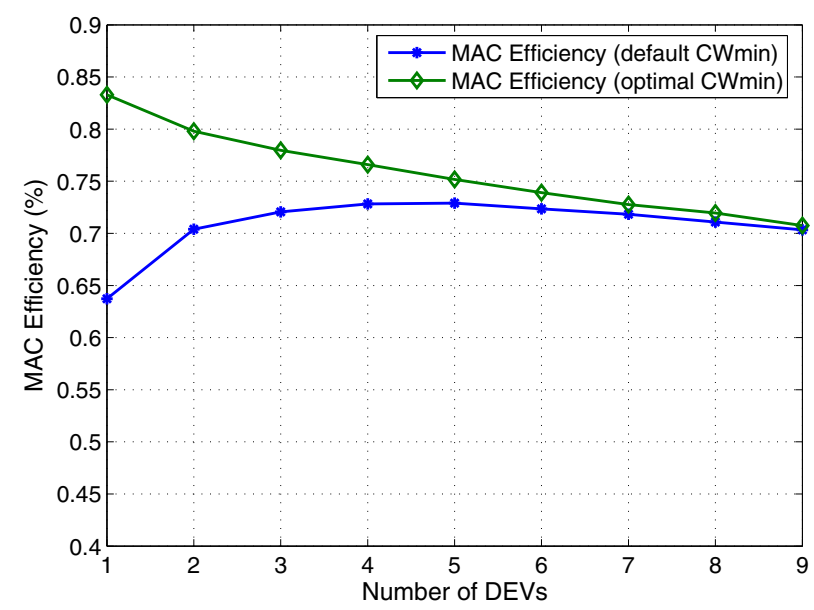

Fig. 10. MAC efficiency vs. number of devices.

DEVs in the network. Note that when there is only one DEV in the network, and CWmin=15, the saturation throughput is about $25 \%$ lower comparing to the maximum achievable throughput. This is because MAC efficiency is reduced due to the extra overhead introduced by random backoff, as illustrated in Fig. 10. MAC efficiency is defined as the ratio of the saturation throughput measured on top of the MAC layer divided by the physical layer data rate.

Figure 11 demonstrates that the access delay increases linearly with the number of DEVs regardless of whether CWmin is set to an optimal or default value. This is because the chance of collisions increases when there are more DEVs in the network.

\section{CONCLUSION}

We proposed and evaluated a directional CSMA/CA protocol for mmWave WPANs. The proposed MAC protocol is highly suited for directional antennas, does not suffer from the deafness problem, and incurs a small protocol overhead. It is evaluated with both analysis and OPNET simulations. We find that when the optimal CWmin is chosen, the throughput degrades gracefully with an increase in the number of DEVs.

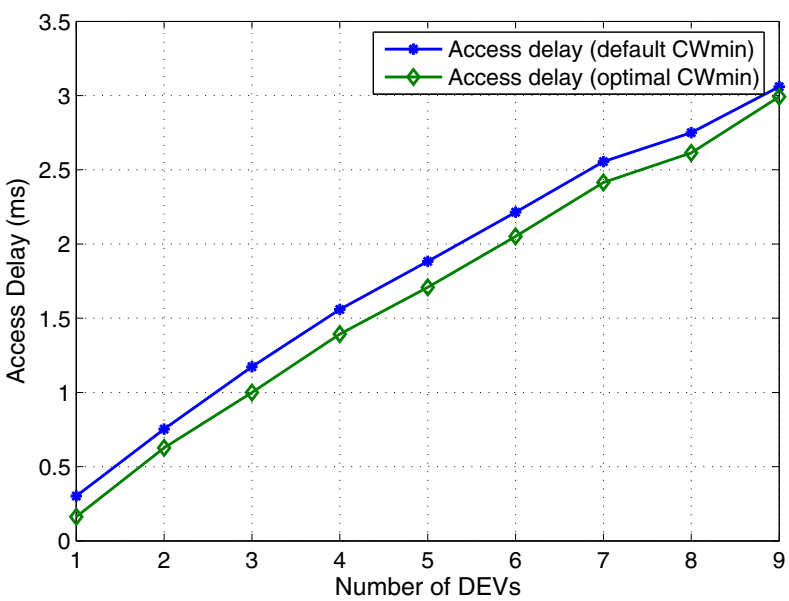

Fig. 11. Access delay vs. number of devices.

Moreover, both our analysis and simulation demonstrate high MAC efficiencies achieved by the proposed directional MAC protocol for mmWave WPANs, especially when the optimal CWmin is chosen.

\section{ACKNOWLEDGMENT}

Shiwen Mao's research is supported in part by the US National Science Foundation (NSF) under Grant ECCS-0802113 and CNS-0855251, and through the Wireless Internet Center for Advanced Technology (WICAT) at Auburn University.

\section{REFERENCES}

[1] ECMA TC48, ECMA standard 387, "High Rate 60GHz PHY, MAC and HDMI PAL," Dec. 2008.

[2] IEEE 802.15 WPAN Task Group 3c Millimeter Wave Alternative PHY (TG3c), http://www.ieee802.org/15/pub/TG3c.html.

[3] X. An and R. Hekmat, "Directional MAC protocol for millimeter wave based wireless personal area networks," in Proc. IEEE VTC-Spring 2008, pp.1636-1640, Singapore, May 2008.

[4] C.-W. Pyo, F. Kojima, J. Wang, H. Harada, and S. Kato, "MAC enhancement for high speed communications in the 802.15.3c mmWave WPAN," Springer Wireless Pers. Commun., vol.51, pp.825-841, 2009.

[5] IEEE 802.15.3 Working Group, "Part 15.3: Wireless medium access control (MAC) and physical layer (PHY) specifications for high rate wireless personal area networks (WPAN)," Draft P802.15.3/D16, 2003.

[6] G. Bianchi, "Performance analysis of the IEEE 802.11 Distributed Coordination Function," IEEE Journal on Selected Areas in Communications, vol.18, no.3, pp.535-547, Mar. 2000.

[7] M. Takai, J. Martin, A. Ren, and R. Bagrodia, "Directional virtual carrier sensing for directional antennas in mobile ad hoc networks," in Proc. ACM MobiHoc 2002, Lausanne, Switzerland, June 2002, pp.183-193.

[8] J. Hsu and I. Rubin, "Performance analysis of directional CSMA/CA MAC protocol in mobile ad hoc networks," in Proc. IEEE ICC'06, June 2006, Istanbul, Turkey, pp.3657-3662.

[9] Y.B. Ko, V. Shankarkumar, and N.H. Vaidya, "Medium access control protocols using directional antennas in ad hoc networks," in Proc. IEEE INFOCOM'00, Tel Aviv, Israel, Mar. 2000, pp.13-21.

[10] T. Korakis, G. Jakllari, and L. Tassiulas, "A MAC protocol for full exploitation of directional antennas in ad-hoc wireless networks," in Proc. ACM MobiHoc'03, Annapolis, MD, June 2003, pp.98-107.

[11] M. Gong, S. Midkiff, and S. Mao, "MAC protocols for wireless mesh networks," in Wireless Mesh Networking: Architectures, Protocols and Standards, Y. Zhang, J. Luo, and H. Hu (Editors), pp.147-182. New York, NY: Auerbach Publications, 2006.

[12] IEEE standard 802.11, "Wireless LAN Medium Access Control (MAC) and Physical Layer (PHY) specification," 2007.

[13] IEEE standard amendment 802.11n, "Enhancements for higher throughput," 2009. 